\title{
Remote multispectral imaging with PRISMS and XRF analysis of Tang Tomb Paintings
}

\author{
Rebecca Lange ${ }^{\mathrm{a}}$, Qunxi Zhang ${ }^{\mathrm{b}}$, Haida Liang ${ }^{*}$

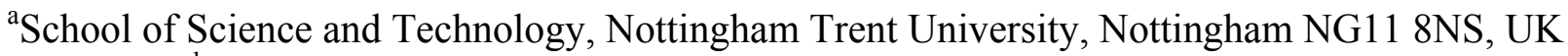 \\ 'Shaanxi History Museum, 91 Xiaozhai East Road, Xi'an 710061, China
}

\begin{abstract}
PRISMS (Portable Remote Imaging System for Multispectral Scanning) is a multispectral/hyperspectral imaging system designed for flexible in situ imaging of wall paintings at high resolution (tens of microns) over a large range of distances (less than a meter to over ten meters). This paper demonstrates a trial run of the VIS/NIR (400-880nm) component of the instrument for non-invasive imaging of wall paintings in situ. Wall painting panels from excavated Tang dynasty (618907AD) tombs near Xi'an were examined by PRISMS. Pigment identifications were carried out using the spectral reflectance obtained from multispectral imaging coupled with non-invasive elemental analysis using a portable XRF.
\end{abstract}

Keywords: multispectral imaging, hyperspectral imaging, remote imaging, infrared imaging, pigment identification, $\mathrm{XRF}$

\section{INTRODUCTION}

The application of multispectral imaging to Western European easel paintings has been an active area of research since the $1990 \mathrm{~s}^{1,2,3,4,5,6,7,8,9,10,11,12}$. Given its non-invasive nature, it has increasingly been applied to the study of manuscripts where in general no sampling of the artefact is allowed ${ }^{4,13}$. A non-invasive imaging technique such as multispectral imaging offers the possibility of virtual sampling at any position on an intact object which is not possible with invasive techniques. Investigations at discrete points give only specific information of the points analyzed but does not give an overall view of the material composition of the object.

In the case of wall paintings which are immobile, a multispectral imaging system has to be portable and capable of high resolution imaging of large areas in situ. PRISMS (Portable Remote Imaging System for Multispectral Scanning) ${ }^{14,15}$ is a multispectral/hyperspectral imaging system specifically designed for flexible in situ imaging of wall paintings at high resolution (tens of microns) over a large range of distances (less than a meter to over ten meters) without the need to lift the imaging system up to the painting. It is potentially useful as an instrument for recording the initial state of the wall paintings as they are being excavated.

The Shaanxi History Museum in Xi'an China has in its collection over 1,000 square meters of wall painting from the Tang dynasty ( $7^{\text {th }}$ to $9^{\text {th }}$ century), which are mostly from excavated tombs of Tang aristocrats. Currently, between three to five Tang tombs with mural paintings are excavated per year in Shaanxi province. The number of tombs excavated has more than doubled in recent years due to increased rate of housing construction. It is particularly important to record the original state of the paintings before they are detached since the rate of deterioration is greatest at the point of excavation.

Initial mock field tests of PRISMS were carried out on wall paintings from tombs of the Tang dynasty paintings in the collection of the Shaanxi History Museum in 2007. Spectral reflectance data obtained from PRISMS were verified with spot measurements using a portable fiber optic spectrometer.

*haida.liang@ntu.ac.uk; phone 441158488056

O3A: Optics for Arts, Architecture, and Archaeology III, edited by Luca Pezzati, Renzo Salimbeni, Proc. of SPIE Vol. 8084, 80840Y · (c) 2011 SPIE · CCC code: 0277-786X/11/\$18 · doi: 10.1117/12.890973 
Non-invasive identification of pigments using spectra obtained from PRISMS were carried out and verified with noninvasive in situ X-ray fluorescence (XRF) analysis of elemental compositions of the pigments.

In this paper, we show an example using a wall painting panel in the collection of the Shaanxi History Museum. The painting was excavated from the Wei family tomb near Xi'an (Chang'an Nanliwang village) dating from the Tang dynasty (Fig. 1).

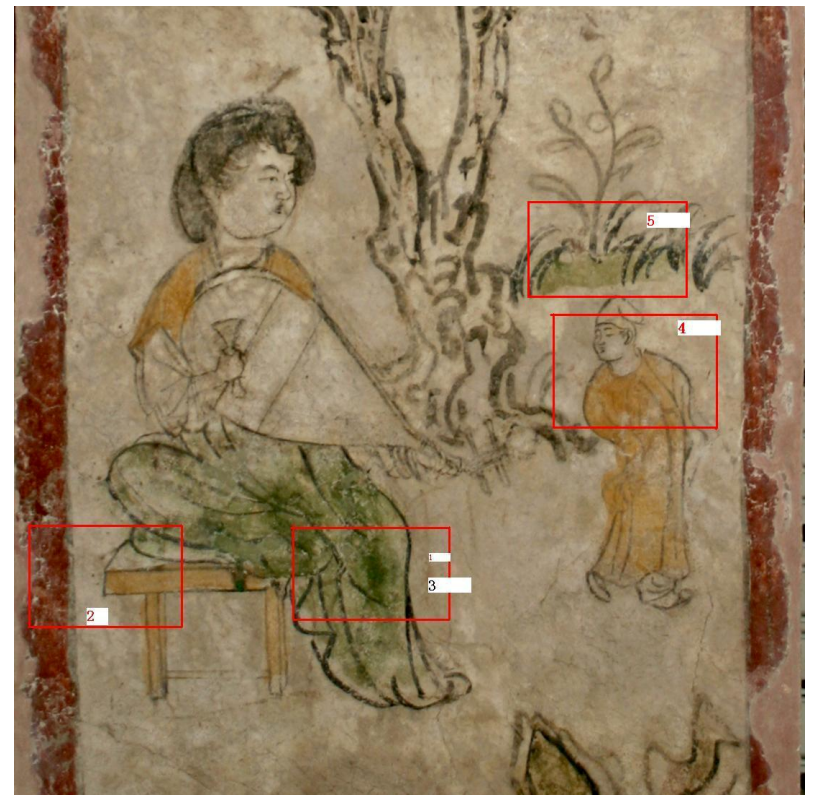

Figure 1. Wall painting panel from the Wei family tomb ( 750AD). The red boxes are regions imaged with PRISMS.

\section{PRISMS}

PRISMS is designed for versatile imaging of wall paintings. It consists of modular components: 1) for imaging remotely at distances $>3.5 \mathrm{~m}$, a telescope is used (Fig. 2a); 2) for close range imaging at distances $<3.5 \mathrm{~m}$, lenses are used (Fig. $2 b)$; 3) for imaging in the wavelength range of $400 \mathrm{~nm}-880 \mathrm{~nm}$, interference filters are used with CCD detectors; 4) for imaging in the short wave infrared range of $900 \mathrm{~nm}-1700 \mathrm{~nm}$, an AOTF (Acousto-Optic Tunable Filter) is used with InGaAs detectors. In this paper only the visible system $(400-880 \mathrm{~nm})$ is used. Spatial resolution of the system is between 12 and 25 pixels per millimeter for distances between $1-\mathrm{m}$ and $10-\mathrm{m}$. The filter system consists of a filter wheel and a set of 10 interference filters between $400 \mathrm{~nm}$ and $880 \mathrm{~nm}$ (Fig. 3). The first nine filters have bandwidth of $40 \mathrm{~nm}$ and are evenly spaced at $50 \mathrm{~nm}$ intervals; the last filter at $880 \mathrm{~nm}$ has a bandwidth of $70 \mathrm{~nm}$. The 14 bit monochrome digital camera, Jenoptik ProgRes MF $^{\text {cool }}$ with peltier cooling has 1360 by 1024 pixels with $6.45 \mu \mathrm{m}$ size pixels. The imaging system stays at ground level during operation. The visible system without the lights was taken from Nottingham to Xi'an via Paris and Beijing in hand luggage by one person. The portability of the system means that it can be taken to remote sites to image large paintings in situ.

\section{CALIBRATION \& VERIFICATION}

\subsection{Standard Calibration}

Calibration of a multispectral imaging system involves both the standard CCD calibration procedure and a spectral calibration procedure to adjust the relative spectral response of the filter channels ${ }^{7}$. Integration time for a target is adjusted per channel such that the maximum intensity in an image is about $\sim 10 \%$ below saturation to ensure the best 
signal to noise in each channel and that the CCD is operating in the linear response range. Dark frames taken at the same integration time as the target frames are subtracted from each frame. Spectral calibration is achieved by imaging a Labsphere spectralon white standard with known spectral reflectance. In this case, only relative spectral calibration is carried out to ensure that the correct spectral shapes are recovered, since pigment identification rather than colorimetry was the main aim in this project. The absolute normalization of the spectrum was not calibrated, i.e. flatfield correct for the spatial inhomogeneity of the illumination was not carried out nor was the Labsphere standard placed at exactly the same position as the painting.

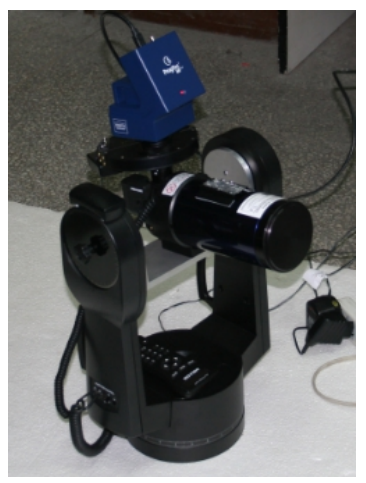

(a)

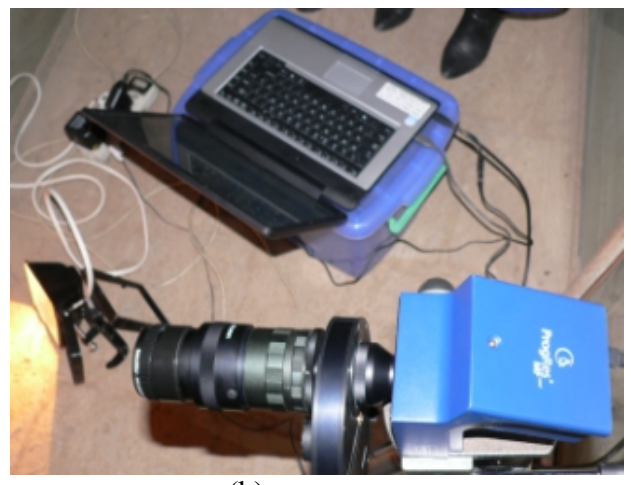

(b)

Figure 2. a) PRISMS with the remote imaging setup using a telescope; b) PRISMS in a close range setup with a lens imaging a painting in situ in the tomb of the Tang Prince Zhanghuai (tomb constructed in 706-711AD).

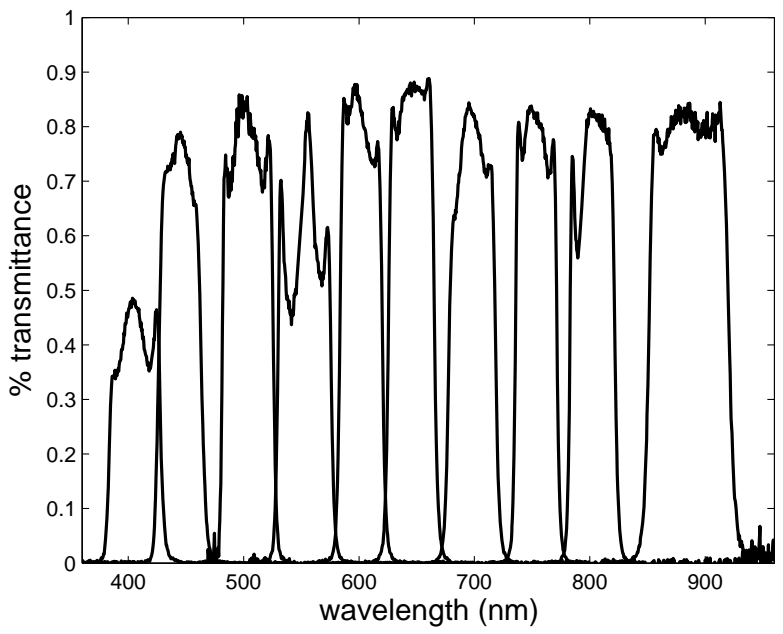

Figure 3. Filter transmittance for the visible system.

In the case of in situ imaging, it is not always possible to have a totally dark background. We have investigated the effects of daylight (through the windows) on the spectral reflectance. Typically the incident illumination is $\sim 400-500$ times that of the ambient light. Figure 4 shows a comparison between the reflectance obtained by subtracting the ambient background (with the imaging illumination turned off but without the lens cap on) compared with that of subtracting a dark frame (with the lens cap on), which indicates that ambient light has little effect on the accuracy of the spectral reflectance measured. 


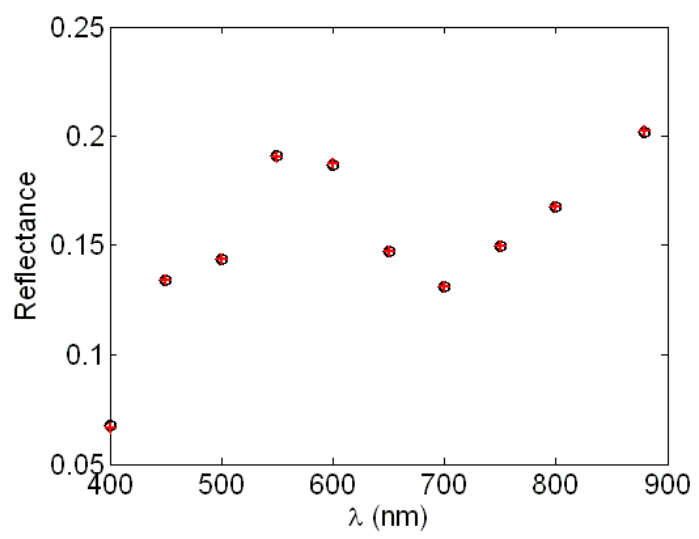

Figure 4. Spectral reflectance with daylight subtraction (black circles) compared with subtracting only the dark frames obtained with the lens cap on (red crosses).

\subsection{Self-Calibration}

This run at the Shaanxi History Museum in 2007 was our first in situ trial run that required transporting the system by plane in hand luggage. We did not take our usual lighting system but instead relied on local lighting supplies. As it was in December and the heating system in the studio was not functioning at the time of the run, some of the images were taken in rather cold conditions and the mechanics of the filter wheel system appeared to be unreliable at times, resulting in some channels giving erroneous readings. Here we show that it is possible to calibrate for the occasional 'bad' channel by using the white background in the same image for self-calibration. Figure 5 shows the spectrometer measured white background spectra compared with the normalized spectra of the white background from a number of PRISMS images of different parts of the wall painting panel. Spectra of the white background in each image was used for self-calibration where a correction factor for the problem channels were obtained by assuming that the white background has a smooth spectrum, which is shown to be the case from independent measurements using a X-RITE-64 spectrocolorimeter.

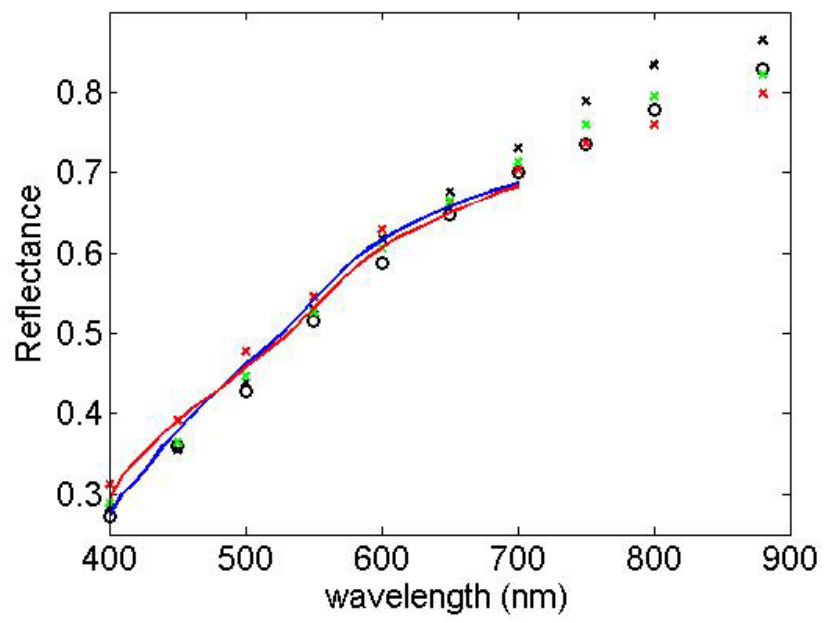

Figure 5. Normalized spectra of the white background in each PRISMS image cube captured on different parts of the same wall painting panel from the Wei family tomb, after correction for problem channels (e.g. channel 2 was incorrect in all images). To correct the channels it is assumed that the white has a smooth spectrum which is verified by the spectrometer data (solid curves). 

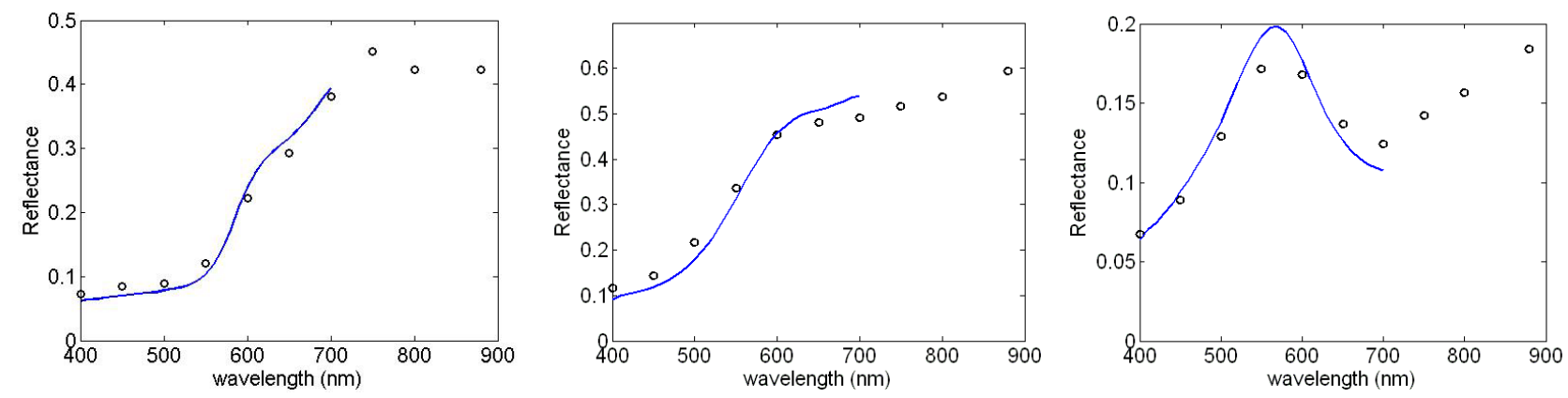

Figure 6. PRISMS spectra compared with direct spectrometer measurement of areas of the wall painting panel from the Wei family tomb. Left: red border; Middle: yellow seat; Right: green skirt. The solid curves are the spectrometer measured spectra and the circles are PRISMS measurements. The PRISMS and the spectrometer measurements are not from exactly the same position. The PRISMS measured spectra have been scaled to the spectrometer measure spectrum, since only relative spectral calibration was carried out for the PRISMS measurements.

Various parts of the wall painting panel were measured directly with a spectrocolorimeter to compare the spectra with those measured by PRISMS which showed that the spectral shape recovered by PRISMS is accurate (Fig. 6).

\section{PIGMENT IDENTIFICATION}

The complementary functions of the two non-invasive methods: large area rapid imaging using multispectral imaging and point based elemental analysis using XRF for pigment identification has long been recognized, however, few studies have combined these two non-invasive techniques ${ }^{16,17}$. Here we combine multispectral imaging with PRISMS and elemental analysis with a SEA200 handheld XRF.

Various reference reflectance spectra were used in this study. Where we have prepared paint samples ourselves or have access to the paint samples, the samples were imaged with PRISMS and analyzed in a similar manner as for the wall painting images.

XRF analysis of the red border in the painting shown in Fig. 1 indicates the presence of Fe and Ca. Figure 8 shows the spectrum of the red paint measured by PRISMS is identified with the reference paint of red earth (from China) in animal glue, which is consistent with the XRF elemental analysis since red earth is mainly iron oxide. The reference paint was prepared in a traditional manner and painted on a mock wall painting substrate.

$\mathrm{XRF}$ analysis found the yellow paint to mainly contain $\mathrm{Pb}$ which suggests a possible pigment identification with litharge. We do not have a litharge paint in our reference sample and the closest we could find online was the spectrum of litharge in linseed oil from the IFAC and OPD database. However, as shown in Fig. 9 it does not fit the spectrum of the yellow paint very well. This could be because of the difference in binding medium and a variation in pigment composition. Further investigation should be carried out by preparing paint outs with litharge (preferably sourced in China) mixed in animal glue or other traditional binding medium most likely to be used in such paintings.

Figure 10 shows that the green in the skirt area does not seem to fit the reference spectrum of malachite despite the XRF results suggesting that it is a copper pigment and therefore most likely to be malachite for Chinese wall paintings. While the reference spectrum is of a malachite (sources in China) in linseed oil paint rather than any of the traditional binding media used for such paintings, it is unlikely that such a change in binding medium is likely to produce a better match in spectrum. Another copper green pigment atacamite is also found in Tang dynasty wall painting (e.g. in Dunhuang). Spectrum of an atacamite pigment powder from Kremer was measured but it also does not have such a high relative reflectance in the red end of the spectrum. Further investigation using atacamite sourced in China prepared with an appropriate binding medium should be conducted. Other possibilities include mixtures of malachite with other pigments. 


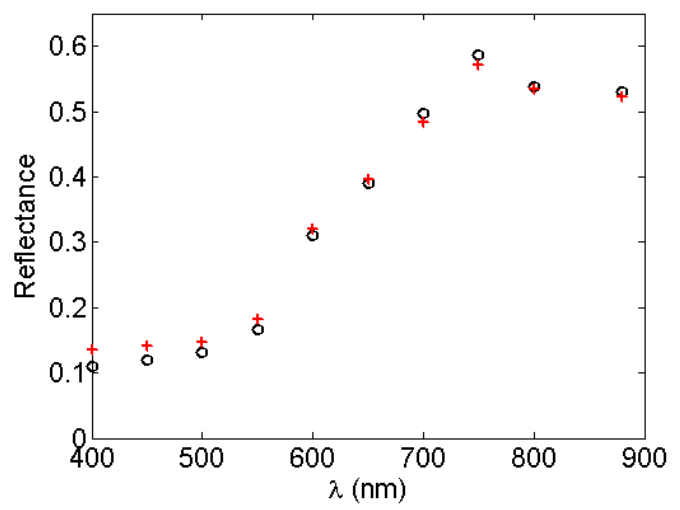

Figure 8. The red border area in the panel from Wei Family tomb is identified with red earth pigment. The circles are the normalized PRISMS measured reflectance spectrum and the red crosses represent the PRISMS measured spectrum of the reference red earth (sourced in China) mixed with animal glue.

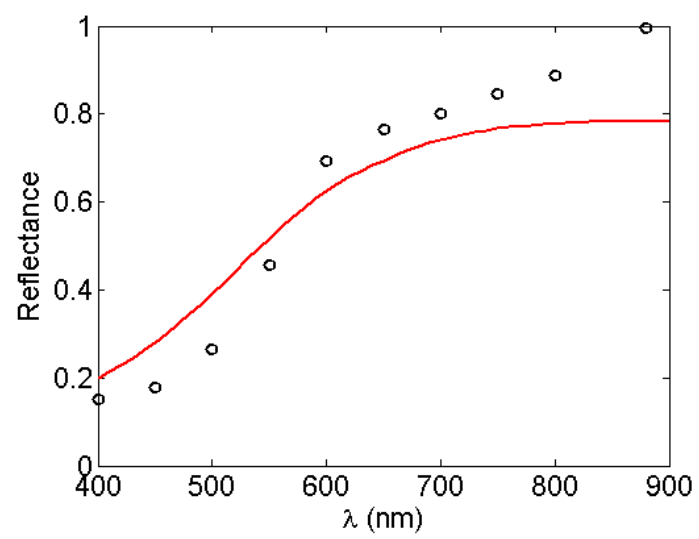

Figure 9. PRISMS measured spectrum of the man's yellow coat in the painting (black circles) compared with reference spectrum from IFAC and OPD database of litharge mixed in linseed oil (http://fors.ifac.cnr.it/).

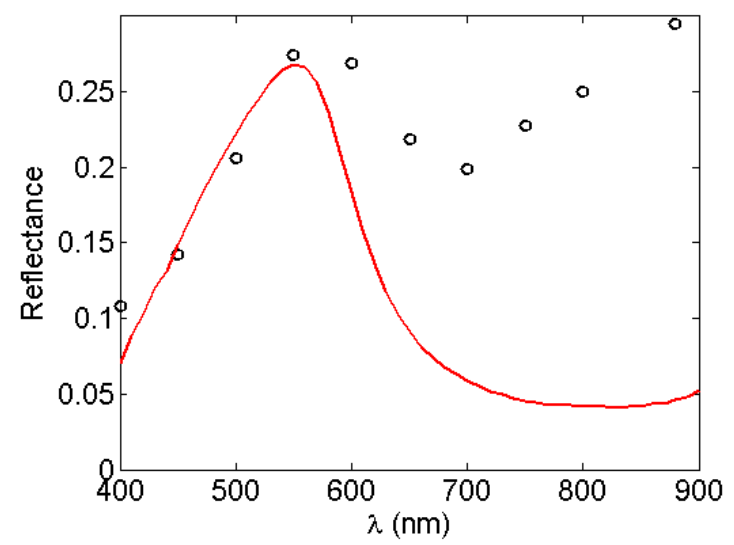

Figure 10. PRISMS measured spectrum of the green in the lady's skirt area (black circles) compared with a reference spectrum of malachite (sourced in China) in linseed oil (red curve). 
Pigment identification using spectral reflectance depends on having an appropriate set of reference paint samples. In addition, effects of pigment concentration, particle size and binding medium can all affect the spectral shape to some extent ${ }^{18}$. Such effects as well as the possibility that the paint may have been degraded must all be taken into account in pigment identification. The advantage of an elemental based analysis such as XRF is that it is not affected by paint degradation and therefore it complements the information from spectral reflectance which is sensitive to the chemical properties of the paint mixture.

\section{CONCLUSIONS}

We have demonstrated through a trial field trip that PRISMS, the portable high resolution multispectral imaging system designed specifically for flexible imaging of wall paintings in situ, is capable of obtaining accurate relative spectral reflectance measurements using simple relative calibration methods. Occasional problems with individual channels can be corrected using a self-calibration procedure by assuming that the spectral shape of the white background of the painting is smooth and monotonic. The imaging system setup is not affected by ambient light which means it can be used for in situ imaging during the day. Pigment identification with PRISMS measured spectra showed that the red pigment in the wall painting panel studied is a perfect match to a red earth pigment (mixed in animal glue), which is also confirmed by XRF elemental analysis. The yellow and green pigment identifications still require further investigation to be conclusive. The study shows that the combination of the two complementary non-invasive techniques adds to our knowledge of the paint composition beyond what each technique alone can give. Multispectral imaging with PRISMS is relatively fast and therefore capable of capturing spectral reflectance on a large scale at spatial resolutions of tens of microns and it is sensitive to the chemical properties of the paint mixture. On the other hand, XRF is capable of specific identification of the elemental composition on a point based analysis with spatial resolution of a few millimeters.

\section{ACKNOWLEDGEMENT}

This work was supported by a UK Engineering and Physical Sciences Research Council (EPSRC) grant (EP/E016227/1). We would like to thank Qingyan Shen of Shaanxi History Museum for useful discussions and encouragement, Tom Vajzovic and Simon Godber for contributions to PRISMS control software development, Kafing Keita for helping with some of the initial tests, Marika Spring for some of the reference paint out and John Cupitt for maintaining the VIPS/nip software (http://www.vips.ecs.soton.ac.uk/). The reference spectrum of a paint mixture of litharge in linseed oil was from the FORS of pictorial materials from 270-1700nm database from IFAC and OPD (http://fors.ifac.cnr.it/).

\section{REFERENCES}

[1] Burmester, A., Cupitt, J., Derrien, H., Dessipris, N., Hamber, A., Martinez, K., Müller, M. and Saunders, D., "The Examination of Paintings by Digital Image Analysis," 3rd International Conference on Non Destructive Testing, Microanalytical Methods and Environmental Evaluation for Study and Conservation of Works of Art, Rome, 201 (1993).

[2] Saunders, D. and Cupitt, J., "Image Processing at the National Gallery: The VASARI Project," National Gallery Technical Bulletin 14, 72-85 (1993).

[3] Baronti, S., Casini, A., Lotti, F. and Porcinai, S., "Multispectral Imaging System for the Mapping of Pigments in Works of Art by Principal Component Analysis," Applied Optics 37, 1299-1309 (1998).

[4] Balas, C., Papadakis, V., Papadakis, N., Vazgiouraki, A. and G. Themelis, "A Novel hyper-spectral imaging apparatus for the non-destructive analysis of objects of artistic and historic value," Journal of Cultural Heritage 4 (1), 330 (2003).

[5] Hardeberg, J., Schmitt, F. and Brettel, H., "Multispectral color image capture using a liquid crystal tunable filter," Opt. Eng. 41, 2532 (2002).

[6] Mansfield, J., Attas, M., Majzels, C., Collins, C., Cloutis, E. and H. Mantsch, "Near Infrared Spectroscopic Reflectance Imaging: A New Tool in Art Conservation," Vibrational Spectroscopy 28, 59-66 (2002).

[7] Liang, H., Saunders, D. and Cupitt, J., “A new multispectral imaging system for examining paintings,” Journal of Imaging Science \& Technology 49, 551 (2005).

[8] Berns, R., Taplin, L., Imai, F., Day, E. and Day, D., "A comparison of small-aperture and image-based spectrophotometry of paintings," Studies in Conservation 50, 253 (2005). 
[9] Delaney, J. K., Walmsley, E., Berrie, B. H. and Fletcher, C. F., "Multispectral imaging of paintings in the infrared to detect and map blue pigments," Sackler NAS Colloquium - Scientific Examination of Art: Modern Techniques in Conservation and Analysis, 120 (2005).

[10] Bonifazzi, C., Carcagni, P., Fontana, R., Greco, M., Mastroianni, M., Materazzi, M., Pampaloni, E., Pezzati, L. and Bencini, D., "A scanning device for VIS-NIR multispectral imaging of paintings," J. Opt. A: Pure Appl. Opt. 10, 064011 (2008).

[11] Daffara, C., Pampaloni, E., Pezzati, L., Barucci, M. and Fontana, R., "Scanning Multispectral IR Reflectography SMIRR: An Advanced Tool for Art Diagnostics," Accounts of Chemical Research 43(6), 847 (2010).

[12] Picollo, M., Bacci, M., Casini, A., Lotti, F., Poggesi, M. and Stefani, L., "Hyperspectral image spectroscopy: a 2-D approach to the investigation of polychrome surfaces," Conservation Science 2007, 162 (2008).

[13] Klein, M., Aalderink, B., Padoan, R., De Bruin, G. and Steemers, T., "Quantitative hyperspectral reflectance imaging”, Sensors 8, 5576 (2008).

[14] Liang, H., Keita, K. and Vajzovic, T., "PRISMS: A portable multispectral imaging system for remote in situ examination of wall paintings," Proc. SPIE 6618, 661815 (2007).

[15] Liang, H., Keita, K. and Vajzovic, T., "PRISMS: remote high resolution in situ multispectral imaging of wall paintings," International Council of Museums, Committee for Conservation (ICOM-CC) Triennial Conference Vol. I, 353-358 (2008).

[16] Casini, A., Lotti, F., Picollo, M., Stefani, L. and Bussegoli A., "Image Spectroscopy Mapping Technique for Non-Invasive Analysis of Paintings," Studies in Conservation 44(1), 39-48 (1999).

[17] Delaney, J., Zeibel, J., Thoury, M., Littleton, R., Palmer, M., Morales, K., de la Rie, E. and Hoenigswald, A., "Visible and Infrared imaging Spectroscopy of Picasso's Harlequin Musician: Mapping and Identification of Artist Materials in situ," Applied Spectroscopy 64(6), 584-594 (2010).

[18] Liang, H., Keita, K., Peric, B. and Vajzovic, T., "Pigment identification with optical coherence tomography and multispectral imaging," Proc. OSAV'2008, The 2nd International Topical Meeting on Optical Sensing and Artificial Vision, 33-42 (2008) 